\title{
Comparison of Post-operative Outcomes of Endoscopic and Microscopic Type 1 Tympanoplasty-A Retrospective Study
}

\author{
Gurumani $S,{ }^{1}$ Valli $R,{ }^{1}$ Deepthy Das ${ }^{1}$
}

\section{Introduction}

$\underline{\text { ABSTRACT }}$

With increasing popularity of Endoscopic middle ear surgeries, a better understanding of the features salient to endoscopic and microscopic tympanoplasty has gained importance. This study aims to compare the results of both. Materials and Methods

It is a retrospective study based on MRD data collected from January 2017 to July 2018 on 40 patients with dry central perforation. 20 patients underwent endoscopic tympanoplasty and other 20 underwent microscopic tympanoplasty. Results of the surgery were compared at the end of 3 months which was based on the outcomes of surgery by means of graft uptake, comparison of pre-op and post-op audiogram readings .

$\underline{\text { Results }}$

Eighteen cases (90\%) of endoscopic surgery had mild CHL while in microscopic surgery 7 cases (35\%) had moderate CHL. Average pre-op $A B G$ was 29.05 dB in microscopic and 22.8dB in endoscopic surgery. Average post-op ABG $12.65 d B$ and 18.4 for endoscopic and microscopic surgery. There was significant improvement in hearing gain in both the surgeries $(p<0.001)$. Graft uptake was found to be have similar outcome.

\section{Conclusion}

Endoscopic tympanoplasty can be a good alternative of microscopic tympanoplasty but needs more training as compared to conventional method.

$\underline{\text { Keywords }}$

Tympanoplasty; Endoscopy; Retrospective Study

$\mathrm{O}$ titis media is a highly prevalent disease in middle ear and poses a serious health problem, more prominently seen in developing countries. Prevalence of COM in rural areas is $16.7 \%$, urban population is $25 \%$ (peripheral areas) and $59 \%$ (Central areas). ${ }^{1}$ Tympanoplasty is a surgical procedure used to eradicate the disease of the middle ear and reconstruct the ear drum. Major disadvantage of the operating microscope is that it provides a magnified image along a straight line which limits the visual field. Therefore the common problems encountered during surgery are in difficulty in visualising the sinus tympani, facial recess, difficulty in removing all the squamous epithelium in the area to be covered by the graft, this leading to subsequent formation of epithelial pearls, development of tympanosclerotic patch, blunting of anterior recess, lateralisation of the graft due to difficulty to visualise anterior quadrant and incomplete placement of the graft. ${ }^{1}$
On the other hand endoscopic ear surgery is an emerging technique. It provides an excellent magnified image with good resolution. And all the problems mentioned earlier are not encountered in this surgery Magnification can be achieved by getting the endoscope closer to surgical field. ${ }^{1,2}$ Major disadvantage of that it is a one handed technique. This became especially cumbersome where there is excessive bleeding. This problem is not encountered in microscopic ear surgeries. Another disadvantage of the endoscope is soiling of scope due to blood. ${ }^{2}$ With increasing popularity of Endoscopic

1 - Department of ENT, Shri Sathya Sai Medical College and Research Institute, Tiruporur, Chengalpet, Tamilnadu

Corresponding author:

Dr Valli R

email: vallivinothkumar11@gmail.com 
Table I: Comparison of graft uptake

\begin{tabular}{|c|c|c|}
\hline & ENDOSCOPIC N(\%) & MICROSCOPIC N(\%) \\
\hline Graft Uptake & $\mathbf{1 9}(95 \%)$ & $\mathbf{2 0}(\mathbf{1 0 0} \%)$ \\
\hline Graft Rejection & 1 & 0 \\
\hline
\end{tabular}

middle ear surgeries, studies to correlate their postoperative outcomes have been undertaken. In this article we have done so by comparing the percentage of graft uptake and post-operative graft uptake.

\section{Materials and Methods}

This retrospective comparative study data was collected from the Medical records department in our hospital of patients who were admitted for unilateral Chronic Otitis Media, who underwent Type 1 Tympanoplasty, who had their pre-operative audiogram taken and who had their post-operative audiogram taken after 3 months of surgery. This was compared, based on their improvement in hearing. Forty (40) patients within the age group of 18-60 years were included in the study, who did not have any complications of chronic otitis media. Twenty (20) consecutive patients of each group (endoscopic and microscopic surgery) were taken up for data collection.

\section{Results}

In a total of 40 patients who had unilateral hearing loss, the mean age group for endoscopic cases was 27 years and microscopic cases was 31 years .

In endoscopic group 95\% patients showed graft uptake while in microscopic surgery all 20 cases $(100 \%)$ had graft uptake.(Table I) The study conducted on 40 patients with the data collected from medical records department between the age group of 16 to 50 years with dry central perforation which was irrespective of the size of the perforation. In endoscopic surgery 12 cases $(60 \%)$ had medium size perforation and 8 cases had small perforation. In microscopic surgery 11 cases $(55 \%)$ had medium size perforation and 9 cases had small perforation. 18 cases $(90 \%)$ of endoscopic surgery had mild CHL while in microscopic surgery 7 cases $(35 \%)$ had moderate CHL. (Table II) Average pre-op ABG was $29.05 \mathrm{~dB}$ in microscopic and $22.8 \mathrm{~dB}$ in endoscopic surgery. (Table III) Average post-op ABG $12.65 \mathrm{~dB}$ and $18.4 \mathrm{~dB}$ for endoscopic and microscopic surgery.(Table

Table II : Comparison of Pre \& Post-op Audiogram

\begin{tabular}{|c|c|c|c|c|}
\hline & \multicolumn{2}{|c|}{ ENDOSCOPIC } & \multicolumn{2}{c|}{ TICROSCOPIC } \\
\hline TYMPANING STATUS & PRE-OP & POST-OP & PRE-OP & POST-OP \\
\hline Normal & 0 & $\mathbf{8}$ & 0 & 2 \\
\hline Mild Hearing Loss & 18 & 12 & 12 & 17 \\
\hline Moderate Hearing Loss & 2 & 0 & 8 & 1 \\
\hline Severe Hearing Loss & 0 & 0 & 0 & 0 \\
\hline
\end{tabular}


Table III:Comparison of Pre-op Air-bone gap in the two groups

\begin{tabular}{|c|c|c|}
\hline A-B GAP & ENDOSCOPIC SURGERY & MICROSCOPIC SURGERY \\
\hline $11-20 \mathrm{~dB}$ & 1 & 3 \\
\hline $21-30 \mathrm{~dB}$ & 5 & 9 \\
\hline $31-40 \mathrm{~dB}$ & 11 & 8 \\
\hline $41-50 \mathrm{~dB}$ & 3 & 3 \\
\hline
\end{tabular}

IV) There was significant improvement in hearing gain in both the surgeries $(\mathrm{p}<0.001)$. (Table V and Table VI) In endoscopic group 95\% patients showed complete graft uptake while in microscopic surgery all 20 cases $(100 \%)$ had complete graft uptake. No complications were noticed during the post op period which was of equal duration for all.

\section{Discussion}

Improvement in air bone gap was statistically significant in both studies. The post operative stay for all the patients was the same. No complications were noticed during the post op period which was of equal duration for all.

In this study the age group according to the inclusion criteria was 18-60 years, which included both sexes, the average age being 29.8 years. This was found to be similar to Tzu-Huang et al. ${ }^{3}$ Patel et al had similar gender distribution as that of our study. It was found that the disease was more common in third decade of life. ${ }^{4}$

We achieved a graft success rate of $100 \%$ with microscopic procedure and 95\% with endoscopic procedure which could be compared to Patel et al and Sinha et al. ${ }^{4,5}$ This difference can be owed to a larger learning curve for endoscopic procedure and also it being a one hand procedure.

Pre-op air-bone gap was $29.05 \mathrm{~dB}$ and $22.8 \mathrm{DB}$ for microscopic and endoscopic procedure respectively. Similar to Huang et al and Patel et al, where the studies had pronounced the same outcome as ours. ${ }^{3,4}$

Post op air-bone gap was $18.4 \mathrm{~dB}$ and $12.65 \mathrm{~dB}$ for microscopic and endoscopic procedures respectively. Outcome and the findings of the study was predominantly based on these results which was similar to Sinha et al and Kumar et al. ${ }^{5,6}$

\section{Conclusion}

In this study of endoscopic assisted and conventional microscopic tympanoplasty techniques, the graft uptake was found to be slightly better in microscopic surgery, while hearing improvement was the same in both the

Table IV:Comparison of post-op Air-bone gap in the two groups

\begin{tabular}{|c|c|c|}
\hline A-B GAP & ENDOSCOPIC SURGERY & MICROSCOPIC SURGERY \\
\hline $0-10 \mathrm{~dB}$ & 8 & 2 \\
\hline $11-20 \mathrm{~dB}$ & 8 & 10 \\
\hline $21-30 \mathrm{~dB}$ & 4 & 8 \\
\hline
\end{tabular}


Table V: Mean Air Bone Gap in both groups pre surgery and at 3 months

\begin{tabular}{|c|c|c|c|c|}
\hline & PRE Sx (MES) & POST Sx (MES) & PRE Sx (EES) & POST Sx (EES) \\
\hline Mean air bone gap in dB & 29.05 & 18.4 & 22.8 & 12.65 \\
\hline Standard deviation & 7.294 & 6.684 & 6.195 & 7.376 \\
\hline P-value & & $\begin{array}{c}<0.0001 \\
\text { (Significant) }\end{array}$ & $\begin{array}{c}<0.0001 \\
\text { (Significant) }\end{array}$ \\
\hline
\end{tabular}

Table VI: Comparison of air bone gap improvement in both groups at 3 months

\begin{tabular}{|c|c|c|}
\hline & POST Sx (MES) & POST Sx (EES) \\
\hline Mean air bone gap in dB & 18.4 & 12.65 \\
\hline Standard deviation & 6.684 & 7.376 \\
\hline
\end{tabular}

surgeries. Endoscopic tympanoplasty can be a good alternative of microscopic tympanoplasty but needs more training as compared to conventional method.

\section{References}

1. Alabbasi AM, Alsaimary IE, Najim JM. Prevalence and patterns of chronic suppurative otitis media and hearing impairment in Basrah city. Journal of Medicine and Medical sciences 2010;1(4):129-33

2. Shoeb M, Vinod G, Samir B, Shashikant M. Comparison of surgical outcomes of tympanoplasty assisted by conventional microscopic method and endoscopic method. Int $\mathbf{J}$ Otorhinolaryngol Head Neck Surg. 2016; 2(4):184-
3. Tzu-Yen Huang, Kuen-Yao Ho, Ling-Feng Wang et al. A comparative study of endoscopic and microscopic approach Type 1 tympanoplasty for simple chronic otitis media. J Int Adv Otol. 2016;12(1):28-31

4. Patel J, Aiyer RG, Gajjar Y, Gupta R, et al.Endoscopic tympanoplasty versus microscopic tympanoplasty in tubotympanic CSOM: A comparative study of 44 cases. Int J Res Med Sci. 2015; 3(8):1953-7

5. Sinha M, Hirani N, Khilnani AK. Comparison of endoscopic underlay and microscopic underlay tympanoplasty:A prospective research at a tertiary care centre in Gujarat. Int J Otorhinolaryngol Head Neck Surg. 2017; 3(4):874-7

6. Kumar D, Thakur VK, Singh SP. A comparative study of endoscopic and microscopic approach tympanoplasty of simple chronic otitis media. IOSR J Dental and Medical Sciences 2016;15(11):101-4. 